\title{
Schedule interaction within contexts set by starting stimuli, background stimuli, and time
}

\author{
KEITH A. CHOQUETTE \\ Harvard Medical School, Cambridge, Massachusetts \\ and Veterans Administration Medical Center, Brockton, Massachusetts \\ and \\ H. WAYNE LUDVIGSON \\ Texas Christian University, Fort Worth, Texas
}

This study was designed to test whether behavioral contrast would emerge between two stimuli that differed in relative favorableness within their respective stimulus contexts, in spite of the fact that the two stimuli signaled the same reinforcement rate. Separate contexts were established via two starting stimuli, each of which signaled the occurrence of two discriminative stimuli with their associated reward schedules, and two background stimuli, which both preceded and accompanied a pair of discriminative stimuli. The subjects were pigeons in a discrete-trial keypress task, in which the schedule, following preliminary phases, became MULT (CHAIN VI 5 (MULT VI $30 \mathrm{sec}$ VI $90 \mathrm{sec}$ )) (CHAIN VI 5 (MULT VI $90 \mathrm{sec}$ VI $270 \mathrm{sec}$ )). A difference in response rate to the stimuli signaling VI 90 sec emerged in all 4 subjects as a result of stimulus context. The effect was most pronounced when only one of the two chains was presented in a daily session. The data indicate that any account of interaction in terms of relative rate of reinforcement that fails to tie this variable to stimulus as well as to temporal context cannot be completely successful.

A hierarchical multiple-schedule task was investigated in this study, using what were termed background and starting stimuli. Background stimuli are actually present during the operation of more than one schedule. In addition to such cues, the context is also set by starting stimuli, which shortly antedate more than one schedule. These cues play a critical role in some theories of discrimination (e.g., Daly \& Daly, 1982; Rescorla \& Wagner, 1972), and analyses of their functions have begun in earnest (Balsam \& Tomie, 1985).

This study was an evaluation of the effect of different contexts set by explicit background and starting stimuli. Such stimuli have been shown to have an important influence on incentive contrast in runway differential conditioning (e.g., by Ludvigson \& Gay, 1967). If different starting stimuli control different subsequent events, then different effective contexts should be created by the different starting stimuli. Similarly, different background stimuli that precede and accompany discriminative stimuli may produce a contextual effect. Therefore, target discriminative stimuli signaling components with the same overall reinforcement rate should nevertheless command different response rates if preceded by different starting

This research was supported in part by the Texas Christian University Research Fund. This study was submitted to Texas Christian University by K. A. Choquette in partial fulfillment of the doctoral degree. Correspondence concerning this article may be directed to Keith A. Choquette, Research Department (116B1), Project CALM, VA Medical Center and Harvard Medical School, Brockton, MA 02401. stimuli and accompanied by different background stimuli. In the present study, the reinforcement conditions associated with background and starting stimuli were varied while we observed response rate in following components for which relative reinforcement rate was constant.

\section{METHOD}

\section{Subjects}

The subjects were 4 adult male Auto-sexer pigeons, 2 about 5 years old at the outset and 2 about 4 years old. The pigeons were housed individually in a continuously illuminated room. All pigeons were maintained at $80 \%$ free-feeding weight, receiving Purina Pigeon Chow after each experimental session and as a reinforcement during the session. The pigeons had continuous access to water in the home cage.

\begin{abstract}
Apparatus
A standard operant box, with a response key in the center of the front wall, was used. The key was dark between trials. The background of each stimulus was either red or green, and the remaining aspects of Stimuli S1, S2, A, B, a, and b were, respectively, a cross, disk, vertical line, horizontal line, square, and triangle. Equipment and scheduling constraints prevented our varying the particular physical characteristics of the stimuli among birds. However, the design of the experiment, involving as it did stimulus reversals and shifts, permitted considerable evaluation of this factor.

Procedure

Pretraining began with 2 days of 30-min habituation placements, which were followed by an automaintenance procedure. The stimuli used for S1 and S2 were, respectively, a cross on red and a disk on green. A daily session of automaintenance consisted of 48 presentations of one of these stimuli, followed by food, with the other stimulus presented the following day. Throughout the experiment, a daily session consisted of 48 such presentations of a stimulus or a chain of stimuli, with each
\end{abstract}


chain presented equally often when more than one chain was scheduled per session.

A type of discrete-trial procedure was also used throughout the experiment, in which a component ended with delivery of a reinforcer, the first and only given during that component. The interval between reinforcement and the onset of the next stimulus was varied on a VT 60 -sec schedule. After 3 days of automaintenance, responsecontingent training commenced, with $\mathrm{S} 1$ and $\mathrm{S} 2$ then signaling, successively, FR1 for 2 days, FI $5 \mathrm{sec}$ for 3 days, and VI 5 sec for 3 days, with the stimuli presented on alternate days as during automaintenance.

Pretraining was concluded with the introduction of two additional stimuli, Stimulus A on a red background and Stimulus b on a green background, both of which served in second links of chain schedules. Stimuli S1 and S2 served in first links of the chains and signaled the second links on VI 5-sec schedules throughout the chain. One chain was presented on one day and the other on the next day. On the first daily session in which one of the stimulus chains occurred, the schedule was CHAIN VI $5 \mathrm{sec}$ VI $15 \mathrm{sec}$.

Both chains were then made CHAIN VI $5 \mathrm{sec}$ VI $30 \mathrm{sec}$. They continued to be presented on alternate days. The schedules remained the same for the S1-A chain, while for the S2-b chain the schedule for the second link became increasingly less favorable on each session in which it appeared, going from VI $60 \mathrm{sec}$ to VI $120 \mathrm{sec}$ to VI $180 \mathrm{sec}$ and finally to VI $270 \mathrm{sec}$. The pigeons remained on CHAIN VI $5 \mathrm{sec}$ VI $30 \mathrm{sec}$ for one session and CHAIN VI $5 \mathrm{sec}$ VI $270 \mathrm{sec}$ for the alternate session until responding stabilized. A Wilcoxon test on daily response rate for each of the most recent 6 days was used as the measure of stability. When no stimulus reached significance at the .1 level, responding was considered stable.

Phase 1 began with the continued daily session, but the overall schedule became hierarchically multiple through the addition of two more discriminative stimuli, each signaling reward on a VI 90-sec schedule (See Table 1). Added to the less favorable context was Stimulus a on a green background. After responding stabilized, Stimulus B on a red background was added to the more favorable context. Across a series of days, therefore, the overall schedule was MULT (CHAIN VI $5 \mathrm{sec}$ (MULT VI $30 \mathrm{sec}$ VI $90 \mathrm{sec}$ )) (CHAIN VI $5 \mathrm{sec}$ (MULT VI $90 \mathrm{sec}$ VI $270 \mathrm{sec}$ )).

In Phase 2, both contexts were presented within a single session. Given the constant 48 trials per session, each chain was thus presented 12 times and each context 24 times. Here, and throughout the remainder of the experiment, the chains were presented randomly with the exception that they occurred equally often, and no chain was presented more than 3 times consecutively.

In Phase 3, Stimuli a and B, both of which signaled VI 90-sec schedules, were interchanged. Thus, Stimulus B, a horizontal bar, occurred on a green background, and Stimulus a, a square, occurred on a red background. The contextual background stimuli were therefore switched for these stimuli, as were the contextual starting stimuli. The same background was maintained within each context, always red following S1 and green following S2. In Phase 4, S1 and S2 were interchanged. Again,

Table 1

Starting Stimuli (S1 and S2),

Discriminative Stimuli (A, B, a, and b)* and Background Stimuli (Red or Green) as Presented in Successive Phases

\begin{tabular}{|c|c|c|}
\hline Phase & Context 1 & Context 2 \\
\hline & $\mathrm{S} 1(5)-\mathrm{A}(30)$ or $\mathrm{B}(90)$ & $S 2(5)-a(90)$ or $b(270)$ \\
\hline $1 \dagger$ & Red-Red & Green-Green \\
\hline & $\mathrm{S} 1(5)-\mathrm{A}(30)$ or $\mathrm{B}(90)$ & $S 2(5)-a(90)$ or $b(270)$ \\
\hline 2 & Red-Red & Green-Green \\
\hline & $S 1(5)-A(30)$ or $a(90)$ & $S 2(5)-B(90)$ or $b(270)$ \\
\hline 3 & Red-Red & $\begin{array}{l}\text { Green-Green } \\
\text { (5) }\end{array}$ \\
\hline 4 & $\begin{array}{c}S 1(5)-B(90) \text { or } b(270) \\
\text { Red-Green }\end{array}$ & $\begin{array}{c}S 2(5)-A(30) \text { or a }(90) \\
\text { Green-Red }\end{array}$ \\
\hline
\end{tabular}

*S1 and S2 were associated with VI 5-sec schedules, while the associated schedules for the discriminative stimuli were: A (VI $30 \mathrm{sec}$ ), B (VI $90 \mathrm{sec}$ ), a (VI $90 \mathrm{sec}$ ), and b (VI $270 \mathrm{sec}$ ). IIn Phase 1, contexts were presented on alternate daily sessions; thereafter, both occurred within a day. the red background occurred along with $S 1$, followed, in this case, by either Stimulus B or Stimulus b, both on a green background; the green background occurred with S2 followed by Stimulus A or Stimulus a, both on a red background.

\section{RESULTS}

The rate of responding to Stimuli a and B is presented in Figure 1. Rates for each subject were separately analyzed with an analysis of variance designed for singlesubject studies (Shine, 1973). Daily response rates served as the basic unit of analysis, but since only one context was present on a given day in Phase 1, comparison of rates occurring on alternate days was accomplished through using pairs of adjacent days. Although multiple analyses increase the probability of attaining significance by chance, most of the comparisons of interest were significant well beyond the .05 level.

Responding to Stimuli a and B was the comparison of major interest, since both stimuli signaled VI 90-sec schedules, but in different contexts. Patterns of reaction to these stimuli, much the same in all subjects, varied with contextual changes across phases. Initially, Stimulus B maintained a response rate the same as, or a little above, that of Stimulus a. Within 3 to 6 days, rates of responding to Stimulus B dropped below those to Stimulus a and remained depressed throughout Phase 1 . Analyses of the difference between rates of responding to Stimuli a and $B$ over their eight presentations yielded significance for all subjects $[F(1,4)=56,55,36$, and 18 , respectively, with $p<.005$ for Subjects $1-3$ but $p=.014$ for Subject 4]. Therefore, there was clear evidence of schedule interaction influenced by the contexts, over and above effects of overall reinforcement rate.

In Phase 2 , in which both contexts occurred within the same daily session, responding to Stimuli a and B became more similar. That is, the higher response rate maintained by Stimulus a compared to Stimulus B at the end of Phase 1 was substantially diminished. In fact, in Subjects 1 and 3, the rates actually converged shortly after the beginning of Phase 2 and then separated in later sessions. Comparison of Stimuli a and B over the last 24 days of Phase 2 for Subjects 1 and 2, and over the last 14 days of Phase 2 for Subjects 3 and 4, revealed significance for Subjects 1,3 , and $4[F(1,12)=59, p<.001, F(1,12)=$ $20, p<.001$, and $F(1,7)=17, p<.004$, respectively].

In Phase 3, the starting stimuli preceding Stimuli a and B were switched, which meant that the former favorable stimulus in one context (Stimulus a) was now an unfavorable stimulus in the other context, and vice versa. This switch resulted in a corresponding switch in response rates for Subjects 2 and 4 . In both cases, Stimulus B sustained the higher rate, beginning on Day 3 for Subject 2 and Day 2 for Subject 4 . Subject 1 continued to respond to Stimulus a at a higher rate than to Stimulus B (see Figure 1), and response rates to Stimuli a and B came together for Subject 3. Analyses of rates of responding to Stimulus a versus Stimulus B in Phase 3 revealed sig- 

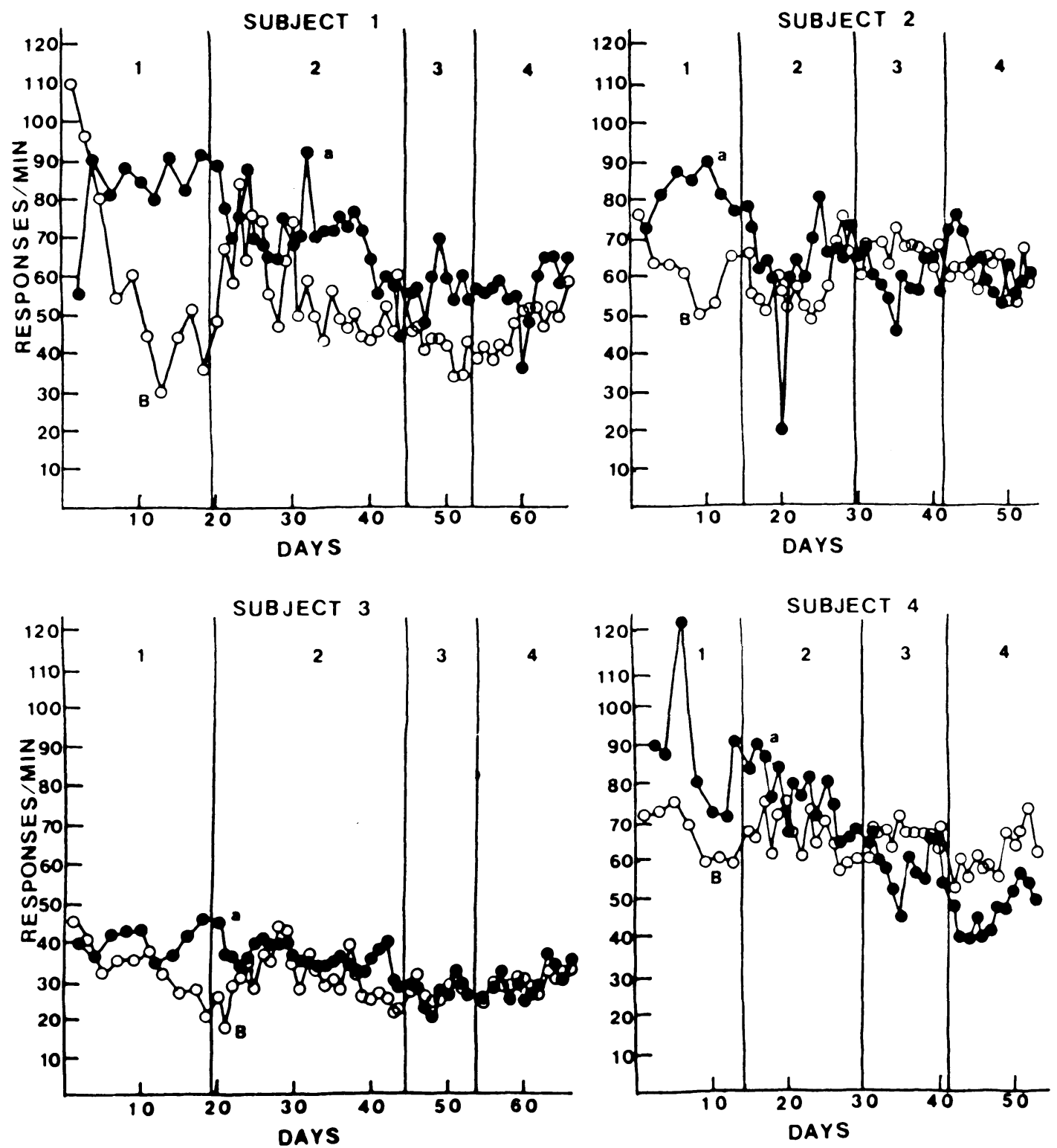

Figure 1. Response rates for daily sessions for individual subjects for Stimuli a and B.

nificant differences for Subjects 1,2 , and $4[F(1,4)=34$, 39 , and $8, p=.005, .001$, and .03 , respectively]. The difference for Subject 1, however, was in an opposite direction from the differences for Subjects 2 and 4 . Therefore, switching contexts for Stimuli a and B appeared to influence responding to these stimuli in all birds except Subject 1 , such that response rate moved away from that sustained by the contrasting schedule in the new context. The switch in contexts for the extreme schedules in Phase 4 produced no noticeable change in response to discriminative stimuli.

To further substantiate the differences in response rate between Phase 2 and the subsequent phases, difference scores were computed by subtracting the daily response rate to Stimulus a from the response rate to Stimulus B. A $t$ test was used to compare the mean difference score for Phase 2 with the mean difference scores for Phases 3 and 4, which differed from Phase 2 due to contextual shifts but not to relative rates of reinforcement. A significant difference was found $[t(3)=4.44, p<.05]$.

\section{DISCUSSION}

The simplest interpretation of the present data is that responding was partly subject to control by distinctive contexts or hierarchical processes within the overall experimental setting. In particular, responding to the signals of the two VI 90 -sec schedules, Stimuli a and B, deviated from overall reinforcement rates in the direction of relative reinforcement rates within the separate contexts. The magnitudes of these effects were small, relative to those of overall relative reinforcement rate. The data from the present study, nevertheless, challenge certain current accounts of schedule interactions-in particular, any account in terms of relative reinforcement rates that does not specify the "context of reinforcement" in terms of the stimulus context. 
Alternative interpretations of the present study appear to be not only more complex than the contextual one but contraindicated by data. For example, one might grant the admittedly potent temporal context effect that occurred in Phase 1 (Stimulus a vs. B), yet resist the inference of contextual effects in later phases by appealing to differences in stimulus similarity. However, in this case, data indicating that contrast is greater with decreasing stimulus similarity (e.g., White, 1986) predict results opposite to those of Phase 2 (not to mention Phase 1). For example, a horizontal line on red is more similar to a vertical line on red than to a triangle on green, but the response difference is greater in the former case. In fact, considering stimulus similarity strengthens the context interpretation, since it appears that contextual effects appeared in spite of stimulus similarity effects working against them.

Although context-specific background stimuli partly controlled interaction processes, this control did not prove very flexible, as is suggested by the slow and sometimes inconsistent reactions to the shifts of Phases 3 and 4 . The general pattern was one of considerable hysteresis. Once the contextual influences on interaction had been generated, there appeared a somewhat inflexible or habitual reaction to the discriminative stimuli.

The contextual influence suggests sensitivity to the relations between associations of contextual and discriminative stimuli; the hysteresis suggests a state conditioned to the discrete discriminative stimuli, at least in some birds. The hysteresis argues against the possibility, entertained by Ludvigson and Gay (1967) some years back, that the interaction process ("frustration" in that case) is a performance outcome of a shift in expectations (or a comparison of expectations), rather than something associated with a stimulus. It also argues against application of the similar comparator hypothesis (Miller \& Schachtman, 1985) regarding the occurrence of conditioned inhibition, as well as scalar expectancy theory (Gibbon \& Balsam, 1981), which could account for differences in response to Stimuli a and $B$ by pointing to the ratio of discriminative associative strengths to context strengths.

It may be important to note that the present study does not preclude the possibility that such comparisons are what initially establish processes that generate interaction-processes that then become associated directly with the discriminative stimuli. Certainly, however, there was no compelling evidence of any recurring comparison process as the basis of interaction. Such a finding might give pause to one impressed with the many demonstrations of cognitive abilities in animals in recent years; it might not be particularly surprising to an S-R psychologist of 20 or more years ago.

\section{REFERENCES}

Balsam, P. D., \& Tomie, A. (Eds.) (1985). Context and learning. Hillsdale, NJ: Erlbaum.

DALY, H. B., \& DALY, J. T. (1982). A mathematical model of reward and aversive nonreward: Its application in over 30 appetitive learning situations. Journal of Experimental Psychology; General, 111, 441-480.

GibBon, J., \& BALSAM, P. D. (1981). The spread of association in time. In C. M. Locurto, H. S. Terrace, \& J. Gibbon (Eds.), Autoshaping and conditioning theory (pp. 219-253). New York: Academic Press.

LUdvigson, H. W., \& GAY, R. A. (1967). An investigation of conditions determining contrast effects in differential reward conditioning. Journal of Experimental Psychology, 75, 37-42.

MilleR, R. R., \& SChachtmen, T. R. (1985). Conditioning context as an associative baseline: Implications for response generation and the nature of conditioned inhibition. In R. R. Miller \& N. E. Spear (Eds.), Information processing in animals: Conditioned inhibition (pp. 51-88). Hillsdale, NJ: Erlbaum.

Rescorla, R. A., \& Wagner, A. R. (1972). A theory of Pavlovian conditioning: Variations in the effectiveness of reinforcement and nonreinforcement. In A. H. Black \& W. F. Prokasy (Eds.), Classical conditioning II: Current research and theory (pp. 64-99). New York: Appleton-Century-Crofts.

SHINE, L. C., II (1973). A multi-way analysis of variance for singlesubject designs. Educational \& Psychological Measurement, 33, 633-636.

WHITE, K. G. (1986). Conjoint control of performance in conditional discriminations by successive and simultaneous stimuli. Journal of the Experimental Analysis of Behavior, 45, 161-174.

(Manuscript received June 13, 1989.) 